\title{
Clinical presentation of neurofibromatosis in indigenous black Africans: Evidence for multidisciplinary team approach to diagnosis and management with emphasis on Ophthalmological perspective.
}

\author{
Olufunmilola OGUN ${ }^{1}$, Olusegun A Adediran ${ }^{2}$, and Gabriel Ogun ${ }^{1}$ \\ ${ }^{1}$ University of Ibadan College of Medicine \\ ${ }^{2}$ University College Hospital Ibadan
}

June 10, 2021

\begin{abstract}
Aim- To describe the pattern of clinical presentation of patients with neurofibromatosis in a homogeneous black African population with emphasis on ophthalmological presentation in a multidisciplinary management setting. Methods- Ophthalmology clinic records from the Department of Ophthalmology and database of the Department of Pathology, both of University College Hopsital, Ibadan, Nigeria were reviewed for cases of neurofibromatosis over a 10-year period (Jan 2010 and Dec 2019 ). Relevant demographic, clinical information, management, complications and patient follow up were extracted from the records and entered into a spreadsheet and analysed. Results - The 34 cases included in this study comprised 19 males (55.9\%) and age ranged from 18 months - 60 years, with a median age of 15 years. The male to female ratio was 1.3:1 with approximately $35.3 \%$ (12 patients) in the paediatric age group. The most frequent reason for consultation was unilateral progressive painless lid swelling (plexiform neurofibroma) often associated with ptosis. There was a positive family history of neurofibromatosis in 9 out of 32 cases $(28.1 \%)$. Café au laît macules were observed in 22 out of $25(88 \%)$ of cases. Typical neurofibromas were present in $84.8 \%$ of the patients. There was no significant difference in prevalence of plexiform neurofibromas with age $(\mathrm{p}=$ $0.05)$ or sex $(\mathrm{p}=0.79)$. Bone and joint abnormalities was present in $17.6 \%$ of the patients. Ophthalmic examination showed conjunctiva changes in 3 cases, prominent cornea nerves in 2 cases. Iris lisch nodules was present in $75.9 \%$ of cases that had documentation, cataract in 2 cases and optic atrophy in 6cases. Three patients had ophthalmic pathway gliomas. Patients were managed by multidisciplinary teams depending on their needs. Conclusion- Multidisciplinary team management is advocated because of the multi-system disorders these patient have and the need for holistic, patient centred care that is of good quality, and sustainable.
\end{abstract}

\section{INTRODUCTION}

Neurofibromatosis (NF) is a relatively common genetic condition characterized by neuroectodermal tumours arising within the skin and organ systems, including the eyes, brain and peripheral nerves ${ }^{1,2} 3-5$. It is autosomal dominant in inheritance with almost complete penetrance and widely variable expressivity ${ }^{6,7}$. Neurofibromatosis is important in ophthalmology because of its propensity for ocular involvement with potentially vision-threatening complications ${ }^{8-13}$. The manifestations and complications of NF are diverse and disease expression varies, even among members of the same family ${ }^{6}$. Although NF can present to virtually any specialty in medicine, ophthalmologists are almost always involved in the management of these patients at some stage of this life-long disease ${ }^{12,13}$. There are many categories of $\mathrm{NF}^{7}$ but the commonest are NF type 1 (NF 1) or classical NF, also known as Von Recklinghausen's disease ${ }^{1,14}$; NF type 2 (NF 2) known previously as central neurofibromatosis, characterized by bilateral acoustic neuroma, now preferentially called vestibular schwannoma ${ }^{15-17}$ and NF type 3 (NF 3) otherwise called peripheral schwannomatosis ${ }^{18,19}$.

Recent advances in molecular biology and neuroimaging, as well as, the development of improved mouse 
models, have helped to elucidate the aetiology of NF1 and its clinical manifestations. Furthermore, these advances have raised the prospect of therapeutic intervention for this complex and distressing disease in the near future ${ }^{2,5}$.

Being a lifelong disorder, the burden shouldered by patients with neurofibromatosis (NF) can be very great, particularly in a resource poor environment ${ }^{20-32}$. Consequently, this should facilitate the recognition of symptoms and signs, promoting early diagnosis and follow-up of these patients. A multidisciplinary approach to management is essential in enhancing the overall quality of life of individuals affected with neurofibromatosis and is strongly advocated for these patients.

This 10-year retrospective review (2010 - 2019) of the patterns of presentation and management of NF in Ibadan, Southwest, Nigeria gives an insight into the clinical and ophthalmic presentation, immediate problems, potential problems and future needs of NF patients, identifying strategies for addressing these issues, with a view to improving the prognosis and quality of life of these patients within a typical African setting.

\section{METHOD}

Neuro-ophthalmology and general ophthalmology clinic records, unit consultation registers and departmental records were reviewed for cases of neurofibromatosis that presented to the eye clinic over a 10-year period (between Jan 2010 and Dec 2019). Cases were also retrieved from the Histopathology records and database of the Department of Pathology, University College Hospital Ibadan for the same period. Relevant demographic and clinical information such as; age at presentation, sex, site of affectation, family history, clinical symptoms, treatment, complications, patients' outcomes and the specific histologic diagnoses were extracted from the records and entered into a spreadsheet, designed for the study. Analysis was performed with the calculation of simple frequencies and proportions. The outcomes were displayed in tables and charts, while relevant clinical images are displayed in figures.

\section{Inclusion and exclusion criteria}

Patients included in this study, were those who met the clinical criteria for the diagnosis of neurofibromatosis; according to the 1988 National Institute of Health (NIH) consensus report ${ }^{3334}$, with or without confirmed histopathological diagnosis of nodular or plexiform neurofibromas or other orbito-ocular or intracranial tumours, associated with neurofibromatosis. On the other hand, cases excluded, were those in whom, there was insufficient clinical evidence to meet the diagnostic criteria for NF, as well as, those with histopathological tissue biopsies supporting a different clinical diagnosis.

\section{Ethical considerations}

This study was performed in compliance with the guidelines of the Helsinki declaration on biomedical research on human subjects. Confidentiality of the identity of the patients and personal health information was maintained.

\section{RESULTS}

A total of 38 cases of neurofibromatosis were documented in records, during the 10-year period, of which 4 cases were excluded from analysis after reviewing their clinical case files. These four cases comprised 2 males and 2 females. Firstly, a 7-month-old female was excluded, based on clinical features of Sturge-weber syndrome associated with unilateral lid swelling from a huge capillary haemangioma, rather than a plexiform neurofibroma. Another 14-month-old female had café au laît skin patches but was excluded when a review of the clinical documentation showed a diagnosis of tuberous sclerosis rather than neurofibromatosis. The third patient excluded, was a 14-month-old male, with a large hypertrophic unilateral facial patch and bony dysplasia initially thought to represent neurofibromatosis, but later diagnosed as Proteus syndrome. Finally, a 58-year-old man documented to have NF1, was excluded for lack of documentation of the diagnostic criteria used and lack of documentation recording ophthalmic assessment or neuro-radiological investigation. The 
prevalence of NIH diagnostic criteria observed, in the remaining 34 cases, which were included in this study, are shown in Figure 1. All cases in this study had features consistent with NF1.

The 34 cases included in this study comprised 19 males (55.9\%) and ages ranging from 18 months - 60 years, with a median age of 15 years, an interquartile range of 19.5 years and the most frequent age at presentation (or diagnosis) was 15 years. The male to female ratio was $1.3: 1(\mathrm{p}=0.35)$ with approximately $35.3 \%$ (12 patients) within the paediatric age group (14 years and below), according to our hospital policy. Presenting visual acuity ranged from Snellen acuity of $6 / 4$ in the better eye to no light perception (NLP) in the worse eye. Although, only one patient presented with poor vision of hand motion (HM) in the better eye, up to a third of patients $(33.3 \%)$ presented with poor vision $<6 / 60$ in the affected eye. All patients were diagnosed with NF 1, based on the NIH diagnostic criteria.

\section{Presenting complaints}

The most frequent reason for consultation was unilateral progressive painless lid swelling (plexiform neurofibroma) often associated with ptosis (33.3\%), other craniofacial swelling including proptosis (plexiform orbito-ocular neurofibroma) was the second most common reason for presenting to the eye clinic $(27.3 \%)$ followed by nodules or plexiform swellings noticed in other parts of the body (12.1\%), while visual impairment $(6.1 \%)$ was the least common reason. Notwithstanding, about 7 patients $(21.2 \%)$ were completely asymptomatic and presented only because they were referred by a physician for ophthalmic evaluation.

\section{Family history}

There was a positive family history of neurofibromatosis in 9 out of 32 cases (28.1\%). The affected first-degree relative was a parent in 7 cases and an older sibling in the remaining 2 . In two of the 7 cases involving a parent, the parents had not been previously diagnosed but had brought their children for consultation and were diagnosed by the consulting physician in the same visit.

\section{General physical manifestations- Café au lâ̂t macules (CALMs)}

The most common systemic sign of neurofibromatosis was the presence of hyperpigmented macules (patches) on the skin; known as Café au laît macules (CALMs) ${ }^{35}$. While most cases had the hyperpigmented (café) spots and patches, very few had the hypopigmented (lait) spots. Even among the patients with hyperpigmentation, this was sometimes difficult to see against the backdrop of their dark complexion (Figure 2a-c). Twentyfive out of 34 cases, had documentation on the presence or absence of CALMs. Café au lait macules were observed in 22 out of $25(88 \%)$ of cases. The distribution was predominantly on the trunk, buttocks and limbs. Identification of CALMs required a general physical examination in most cases, as they are not readily visible. Axillary and inguinal freckling was very uncommon, seen in only 2 cases (5.9\%). We observed multiple crops of hyperpigmented macules measuring about $2-3 \mathrm{~mm}$ in diameter, on the palms of one patient.

\section{Neurofibromas}

The typical plexiform (84.8\%), cutaneous and subcutaneous $(71.4 \%)$ neurofibromas were the second and third most prevalent systemic manifestations observed. These are the most striking clinical manifestations of the disorder and are more noticeable than the CALMs. The distribution of plexiform neurofibromas is shown in Figure 3. The commonest site of occurrence for plexiform neurofibroma was in the craniofacial region, affecting the lids in half of all cases and other parts of the head and neck in another $25 \%$. Figure 4 shows typical histopathological finding of plexiform neurofibroma from the eyelid of an 11 year old female. The forearms and upper limbs were the least common locations (3\%). A plexiform neurofibroma of the nose is shown in Figure 5a and proptosis from an orbitocranial neurofibroma in Figure 5b. There was no significant difference in prevalence of plexiform neurofibromas with age $(\mathrm{p}=0.05)$ or sex $(\mathrm{p}=0.79)$.

\section{Bone and joint abnormalities}

Bone dysplasia was identified in $6(17.6 \%)$ cases: involving the sphenoid bone; the tibia; the spine; as well as, pseudoarthrosis of the interphalangeal joints of the phalanges (Figure $5 \mathrm{c}$ and d). Two of these, had involvement of long bones (including bowing of the tibia shown in Figure 5e), two cases had scoliosis, one 
of these had kyphosis, while absence of the sphenoid bone (sphenoid dysplasia) was seen in 2 cases on neuroimaging (cranial CT scan). A case of sphenoid aplasia is shown in association with an orbito-cranial neurofibroma in Figure $5 \mathrm{f}$.

\section{Neurological and cognitive assessment}

While none of the patients had formal neuro-cognitive assessment, at least 2 cases (5.9\%), were reported to have significant difficulties with school performance that set them apart from their peers. However, it was also observed that this information was not routinely sought during clinical assessment; as there was no documentation of neuro-cognitive performance in majority of cases.

\section{Ophthalmic manifestations of neurofibromatosis}

\section{Ocular adnexa and anterior segment}

Lids and periorbital region: Plexiform neurofibroma was diagnosed in $84.8 \%$ of cases (i.e. 28 out of 33 patients), of which, the majority (11 out of 28) involved the upper eyelid. This was associated with drooping of the upper lid (ptosis) and s-shaped deformity of the lid margin. In 8 of these 11 cases $(72.7 \%)$, the affected eye showed some degree of amblyopia at presentation. Other adnexal manifestations included the presence of cutaneous and subcutaneous neurofibroma nodules on the face, head and neck, as well as plexiform neurofibromas of the orbit, causing proptosis in 4 cases (Figure 5 a \& b). In one of these cases, the orbital swelling was accompanied by intracranial extension as a result of a defect in the lesser wing of the sphenoid (sphenoid dysplasia), shown on computerized tomography (CT) scan of the brain and orbit (same case described above, Figure 5f). Proptosis was associated with varying degrees of lagophthalmos and limitation of ocular motility. However, the lagophthalmos was not associated with corneal exposure or impaired Bell's reflex in either case.

Conjunctiva: Conjunctival changes were observed in 3 cases, comprising neurofibroma in 1 patient (2.9\%), brownish discolouration in another case and ciliary injection in the third case.

Cornea: Prominent corneal nerves were documented in only 2 cases $(5.8 \%$ ), and a corneal opacity (adherent leucoma) was recorded in one patient. One child had ipsilateral glaucoma, in the eye with plexiform neurofibroma of the lid.

Iris (Lisch nodules): Iris harmatomas (Lisch nodules), shown in Figure 6a and b, were found on slit lamp examination, in 22 out of 29 cases $(75.9 \%)$ constituting the second most common ocular sign. Lisch nodules were documented in 2 children, aged 5 years. One patient had segmental iris atrophy.

Pupils: Pupillary examination revealed normal shaped pupils in most cases. However, pupillary reactions were abnormal, showing afferent pupillary defects, in 6 cases. Ectropion uvea was not documented in any case.

Lens: Cataract was present in two cases, although these were not the posterior subcapsular type, often associated with NF type 2 .

Posterior segment

Choroid, Retina and Vitreous: The retina, choroid and vitreous were normal in almost all cases except for a middle-aged man, who had bilateral chorioretinal atrophy and pigmentary degeneration in a distribution akin to retinitis pigmentosa. However, he denied any symptoms of night blindness (nyctalopia). Furthermore, one 10-year-old boy had unilateral total retinal detachment (in the eye, previously described above, with ciliary flush).

Optic nerve: There was optic atrophy in 6 cases. Of these, one patient had bilateral diffuse optic atrophy (Figure $6 \mathrm{c}$ and $\mathrm{d}$ ) associated with optic-hypothalamic pathway glioma (Figure $6 \mathrm{e}$ and $\mathrm{f}$ ). There was one case of glaucomatous cupping. Moreover, one patient (i.e. the same patient with unilateral retinal detachment) had papilloedema (in the contralateral eye) and was found to have an intracranial (intraventricular) glioma on neuro-imaging ${ }^{27}$. There were no cases of optico-ciliary shunts. 


\section{Optic pathway glioma}

Three patients had optic pathway gliomas (8.8\%). There were 2 cases of optic nerve gliomas, one of which appeared malignant, while 1 case had optic-hypothalamic pathway glioma (Figure 6e and f).

\section{Radiological and non-radiological imaging}

Six cases had neuro-imaging. Of these, 3 cases had cranio-orbital CT scans, 2 cases had magnetic resonance imaging (MRI) of the brain and 1 case had MRI of the spine. Three cases had orbito-ocular ultrasonography (B-scan); retinal detachment was diagnosed in two cases, while the third B-scan showed a resolving haematoma, in a patient with suspected plexiform neurofibroma and a recent history of trauma. One patient with scoliosis, had plain radiograph of the spine. Majority of cases however, did not perform radiological investigations. Neuro-radiological diagnoses are listed in Table 1. More advanced forms of ophthalmological imaging such as Optical coherence tomography (OCT), indocyanine-green and fluorescein angiography, as well as, infrared and near-infrared reflectance imaging are not available in our environment and therefore were not used.

\section{Intervention, outcome and follow-up}

Five patients had surgical excision, comprising excision biopsies for 4 plexiform neurofibromas of the lid and neurosurgery for the excision of an intracranial, intraventricular glioma. All patients were successfully followed up for at least 6 weeks postoperatively. Surgery involved the ophthalmologist alone or the ophthalmologist as a member of an interdisciplinary surgical team and neurosurgeons alone in 1 case. A 29-year-old male with a plexiform spinal neurofibroma, had successful surgical resection and physiotherapy. He was managed in a multidisciplinary team. At least 6 cases recommended for neuro-radiological imaging and surgery, were unable to have surgery and were subsequently lost to follow-up.

\section{DISCUSSION}

The earliest clinical study of neurofibromatosis (NF), among Nigerian patients, was reported by Ademiluyi and Ijaduola, in $1988^{21}$. The paper described the clinical features of 14 children with NF, seen over a 6 -year period. Ademiluyi and Ijaduola, made the important observation that in blacks, the typical CALMs consist more of hyperpigmented macules (café), with very few hypopigmented patches (laît) preferably referred to as Café sans laît spots in black patients ${ }^{21}$. Their observation is corroborated in this current study, where it was observed that, hypopigmented macules are very rare, in black African patients, in comparison to the hyperpigmented spots, some of which are also difficult to identify, against the backdrop of their darkskinned complexion (Figure 2b). This lends credence to Freidman's earlier assertion that the intensity of pigmentation of CALMs are dependent on the degree of pigmentation of the background skin ${ }^{6}$. Therefore, a preponderance of dark/ hyperpigmented macules (café sans lait macules, CSLM) is to be expected in black African patients. The distribution of CALMs (or CSLMs) is widespread over the body, although they are reported to spare the scalp, eyebrow areas palms of the hands, and soles of the feet ${ }^{6}$. However, in this study, we observed hyperpigmented CALMs on the palms of one patient simulating "freckling" (Figure 5d) and multiple hyperpigmented macules on the scalp in another patient (Figure 2d).

Interestingly, despite the fact, that CALMs (or preferably CSLMs) are the most common feature of NF among our patients, they are the least concerning to them and are often overlooked as "birth marks", and the presence or proliferation of CALMs (CSLMs) does not prompt patients to seek consultation ${ }^{1,7}$. Moreover, despite the presence of prominent cutaneous, and less commonly, subcutaneous neurofibromas, which are more striking and not easily ignored, many patients still fail to seek consultation, even for these swellings; especially if there is also a parent or sibling with similar lesions. While cutaneous neurofibromas tend to be painless and slow-growing, subcutaneous nodules may become painful ${ }^{6}$.

Plexiform neurofibromas on the other hand, do seem to prompt medical consultation, probably because of their progressive increase in size, propensity for disfigurement and their tendency to cause pressure symptoms or, in the case of lid lesions, impairment of vision ${ }^{22}$. Plexiform neurofibromas can be nodular or diffuse ${ }^{6}$. 
Consequently, it is not surprising that progressive increase, in size of a lid lesion, was the commonest reason for consultation in our study.

We recorded a much higher prevalence of plexiform neurofibromas (84.8\%) in our present study in comparison to another study on NF1, carried out in Ilorin, Nigeria ${ }^{24}$, which reported only $8.2 \%$ plexiform neurofibromas. This may be attributed to the worrisome cosmetic effects of facial and orbito-ocular masses or, to the tendency for plexiform neurofibromas of the lid to cause ptosis, or compress the globe, resulting in visual impairment; thereby prompting early presentation.

Ophthalmic manifestations of NF are somewhat under-reported in African patients and in African literature. For instance, Odebode et al. reported ophthalmological examination in only 5 out of 98 patients $^{24}$. Nevertheless, Lisch nodules have also been reported in individual case reports of African patients with NF1 ${ }^{36,37}$. The Lisch nodules in our study were raised, light yellow, nodules on the iris, which demonstrated increasing prevalence and preponderance with age, similar to descriptions in literature ${ }^{6,13,38,39}$. Our youngest patients with Lisch nodules were aged 5 years, which is relatively early. Lisch nodules characteristically occur after the first decade of life ${ }^{6}$ although Lisch nodules have been described in a child as young as 2 years of age ${ }^{13}$. Prevalence of Lisch nodules correlates with age and is a highly specific sign for diagnosis, even in asymptomatic relatives, but has no other clinical significance ${ }^{6,8,13}$. It is a valuable practice; to examine accompanying relatives in all suspected cases of neurofibromatosis as the presence of Lisch nodules alone is enough to establish a positive family history $4,7,8,38$.

Other anterior segment manifestations of NF are much less common than Lisch nodules and include, glaucoma, ectropion uvea, cataract, conjunctival neurofibroma, prominent corneal nerves and optic pathway glioma. While we documented glaucoma in one case, which was ipsilateral to a large plexiform lid neurofibroma, there was no case with ectropion uvea. We documented full cataract in two cases, however, it is possible that these were incidental. Posterior subcapsular cataract has been documented in association with NF type 2, and is an important diagnostic criterion in the presence of a unilateral acoustic neuroma ${ }^{40,41}$. We also had a case of conjunctival neurofibroma, which is rare ${ }^{40}$. Conjunctival neurofibroma may be associated with significant keratinization of the overlying epithelium due to exposure and irritation. We observed prominent corneal nerves in two cases, corneal nerves become prominent as they hypertrophy in the corneal stroma ${ }^{13}$. They are visible only within 1-2 $\mathrm{mm}$ of clear cornea beyond the limbus and can easily be missed if not specifically sought.

Optic pathway gliomas and intracranial tumours, though rare, occur with a higher frequency in patients with NF, than the general population ${ }^{1,42}$. The prevalence of optic pathway gliomas in this study was $8.8 \%$. However, compared with other reports from Nigeria, this is the only study which reports the presence of these tumours ${ }^{10,20-26,28-30,36,43-51}$. We also identified a rare primary brain glioma in one child with sporadic NF1 ${ }^{27}$. The child had not previously been diagnosed as a case of NF and had presented as a painless, red eye, with loss of vision. Suspicion of an underlying neuro-cutaneous disorder, was raised by the large hyperpigmented forehead patch (Figure 2d), which led to a search for the other diagnostic signs. The child had CALMs (Figure 2c), Lisch nodules and a plexiform neurofibroma of the ankle, which had been excised twice before, but never sent for histological examination. It was therefore important, for the ophthalmologist, to recognize the significance of papilloedema in his contralateral eye and insist on neuroimaging, which identified the intraventricular tumour. Despite the large size, the child had no history of headaches, though he was having difficulties with his performance at school. This emphasizes the importance of clinical observation and detailed examination in the African setting, because clinical history is often sparse. Another case that reiterates the above fact is the 17 year old presenting with poor vision and bilateral optic atrophy. His mother had all the signs of NF1, but had never been diagnosed. He had received several spectacle prescriptions and was referred for low vision aids, without a diagnosis. It was the presence of Lisch nodules on slit lamp examination of the patient that prompted a search for other diagnostic signs, which revealed cutaneous nodules, Lisch nodules (Figure 6b) and CSLMs in the mother, sufficient to make a diagnosis of NF1 (in the mother) and a positive family history of NF, for the patient. Notably, some of the CSLMs were barely perceptible in the 17 year old (Figure 2b, arrow). However, it was in the context of this background NF that the optic atrophy (Figure 
$6 \mathrm{c}$ and d) took on new significance; prompting an insistence on neuroimaging and the eventual diagnosis of optic-hypothalamic pathway glioma (Figure 6a and b).

\section{Benefit of interdisciplinary or multidisciplinary teams and management}

\section{Plexiform neurofibroma}

Plexiform neurofibromas can be difficult to manage especially the diffuse forms, because they tend to be infiltrative in their growth ${ }^{52}$; as such, complete surgical excision is difficult or impractical, therefore recurrence is common ${ }^{3,13}$. One, out of the 5 patients who had surgery, in our study, developed recurrence within 5 months of surgical excision. In a longitudinal study of plexiform neurofibromas, Tucker et al observed a trend towards larger plexiform masses in children, with a higher growth rate in tumour size, than adults ${ }^{3}$. There was no significant age or gender difference in the prevalence of plexiform neurofibromas in our study. Although 1 of our cases returned with significant growth and pain in a craniofacial plexiform neurofibroma, after 7 years' loss to follow up, majority of our patients are unable to afford serial monitoring of plexiform neurofibromas using MRI. Plexiform neurofibromas require multidisciplinary team care due to their complexity and invasiveness. As surgical excision may be extensive, plastic reconstructive surgery may be required at the same sitting or in stages. Neurosurgery may be required to intervene for orbital plexiform neurofibromas, which invade the intracranial cavity, as was the case in almost half of our cranio-facial neurofibromas. In one case, the neurofibroma invaded the region of the facial nerve, causing facioparesis. This case should benefit from the expertise of a maxilla-facial surgeon in a multidisciplinary team setting without additional logistic delays such as scheduling fresh outpatient consultations, in a different clinic or additional cost. In our study, there was a tendency for patients to fail to return for follow-up (becoming lost to follow up) when they were referred to other specialty surgical clinics for review or additional consultation. It is possible that the additional cost of including an additional surgical team, including the logistics and delays, that may occur with scheduling joint surgeries, may have discouraged them. This would be avoided if a multidisciplinary team were already established and working seamlessly as a single unit sharing information and resources. Surgical excision remains the main form of management available to our patients as recent therapies such as interferon alpha$2 \mathrm{~b}$ and the tyrosine kinase inhibitor, imatinib are not accessible ${ }^{13}$.

\section{Amblyopia, glaucoma and undiagnosed refractive errors}

Plexiform neurofibromas of the lid may be accompanied by ptosis, obscuring vision and predisposing to deprivation amblyopia ${ }^{13}$. The pressure of the lid, on the globe, may cause varying degrees of meridional astigmatism, curvature myopia or other refractive errors, which may remain uncorrected, leading to anisometropic amblyopia if not specifically screened, identified and treated ${ }^{13}$. Though rare, ipsilateral glaucoma has been identified in association with a palpebral plexiform neurofibroma. The prevalence of glaucoma in association with plexiform neurofibroma can be as low as $1-2 \%{ }^{13}$ or as high as $23 \%{ }^{40}$ in some reports. We observed glaucoma in only one case $(2.9 \%)$. Current guidelines for the follow-up of NF1 patients, recommend an annual assessment for glaucoma ${ }^{4}$. Regular assessment for refractive errors and amblyopia should be performed in patients having serial monitoring of a palpebral or craniofacial plexiform neurofibroma, to inform the need for surgical intervention.

\section{Neuro-cognitive impairment}

Although developmental psychologists are in very limited supply in the typical African setting, a multidisciplinary team for the management of NF should include not just clinicians and surgeons, but also parents and educationalists (including teachers, educational psychologists, developmental psychologists) as well ${ }^{35}$. Poor school performance was identified in $2(5.8 \%)$ of our cases. In our analysis, we used school performance as a surrogate for neuro-cognitive impairment, since this was not specifically tested. However, this low prevalence should not be misleading. When specifically screened, neuro-cognitive impairment has been found in up to $40 \%$ of cases of NF $1^{53}$. In this study, we observed the failure to assess or document relevant information about cognitive function in more than $90 \%$ of cases. Cognitive impairment, in majority of NF cases, is often mild ${ }^{54}$. We therefore suggest that school teachers or administrators could be trained, as part of a multidisciplinary team for management of NF, to identify and refer individuals with consistently poor performance for their 
age, for medical and ophthalmic screening to exclude NF and other neurological complications. Furthermore, school age patients on follow up for NF could have annual assessment of their cognitive function to facilitate early diagnosis of impairments ${ }^{54}$.

Optic pathway glioma and intracranial tumours

Management of optic pathway gliomas is controversial and challenging because their clinical manifestation varies widely, from a relatively benign clinical course, to significant morbidity ${ }^{4,13,42}$. The management of optic pathway gliomas involves the spectrum from observation to surgery, chemotherapy and radiotherapy. As it is so rare among black Africans, the best strategy is as yet undetermined. Though, observation requires serial neuro-imaging, this in itself, is a financial burden to many patients. Serial visual evoked potentials, being much cheaper, have been advocated by some authors, but this is also not readily available and also has limitations ${ }^{42,55}$. Following diagnosis, the patient is either managed by the oncologist, orbital surgeon (if discrete) or referred to the neurosurgeon. Existing guidelines are unclear as to the subspecialty that is primarily responsible. Where a multidisciplinary team exists, the expertise may be found within the team or an invitation is extended to a specialist to join the group. A similar situation exists with a patient who has an intracranial pathology in the setting of a multisystem disorder such as NF.

\section{CONCLUSION}

Medical practice has become highly specialized and though, with subspecialization, physicians have become highly skilled in specific areas. This is largely to the advantage of patients with disorders limited to specific parts of the body system. However, patients such as those with NF, who have multi-system disorders, may be caught between the cracks, especially in Africa, where the cost of treatment is still largely borne outof-pocket. Therefore, the logical solution to providing high quality healthcare to such patients is to provide treatment through multi-disciplinary teams. A multidisciplinary team may have to cut across traditional medical boundaries to involve other professionals and in some-instances even non-medical social groups, to ensure the delivery of holistic, patient-centered healthcare that is affordable, sustainable and of a quality that assures good outcome and promotes a reasonable quality of life.

Finally this study has the limitation of being a retrospective study with the occurrence of missing data/ information. For the instances of missing data, this was taken into account at the stage of data analysis.

\section{Acknowledgement: None}

\section{Funding: None}

\section{References}

1. Lynch TM, Gutmann DH. Neurofibromatosis 1. Neurologic clinics.2002;20(3):841-65.

2. Lobbous, Bernstock, Coffee, et al. An Update on Neurofibromatosis Type 1-Associated Gliomas. Cancers. 2020;12(1):114. doi: 10.3390/cancers12010114.

3. Tucker T, Friedman JM, Friedrich RE, Wenzel R, Funsterer C, Mautner VF. Longitudinal study of neurofibromatosis 1 associated plexiform neurofibromas. Journal of Medical Genetics. 2008;46(2):81-85.

4. Ferner RE, Huson SM, Thomas N, et al. Guidelines for the diagnosis and management of individuals with neurofibromatosis 1. Journal of Medical Genetics. 2006;44(2):81-88.

5. Ward BA, Gutmann DH. Neurofibromatosis 1: From lab bench to clinic.Pediatric Neurology. 2005;32(4):221-228.

6. Friedman JM. Review Article : Neurofibromatosis 1: Clinical Manifestations and Diagnostic Criteria. Journal of Child Neurology. 2002;17(8):548-554.

7. Riccardi VM. Neurofibromatosis: Clinical Heterogeneity. Year Book Medical Publishers, Inc.; 1982. 
8. Lubs MLE, Bauer MS, Formas ME, Djokvik B. Lisch nodules in Neurofibromatosis Type1. New England Journal of Medicine.1991;324(18).

9. Saxena RC, Saxena S. Lisch spots in neurofibromatosis type 1.Indian J Ophthalmol. 1991;39(4):179-180.

10. Ehigiamusoe FO, Ogbeide E. Neurofibromatosis with pulsating exophthalmos. Port Harcourt Medical Journal. 2017;11(2):99.

11. Gaonker CH, Mukherjee AK, Pokle M. Involvement of the eye and orbit in neurofibromatosis type 1. Indian J Ophthalmol. 1992;40:2-4.

12. Lewis RA, Riccardi VM. Von Recklinghausen neurofibromatosis: Incidece of iris harmatoma. Ophthalmology. 1981;88(4):348 - 354.

13. Kinori M, Hodgson N, Zeid JL. Ophthalmic manifestations in neurofibromatosis type 1. Survey of Ophthalmology.2018;63(4):518-533.

14. Ferner RE. The neurofibromatoses. Pract Neurol2010;10:82-93.

15. Martuza RL, Eldridge R. Neurofibromatosis 2. New England Journal of Medicine. 1988;318(11):684-688.

16. Evans DG, Moran A, King A, Saeed S, Gurusinghe N, Ramsden R. Incidence of Vestibular Schwannoma and Neurofibromatosis 2 in the North West of England over a 10 year period: higher incidence than previously thought. Otol Neurotol. 2005;26(1):93-97.

17. Evans DGR, Baser ME, O'Reilly B, et al. Management of the patient and family with neurofibromatosis 2: a consensus conference statement.British Journal of Neurosurgery. 2005;19(1):5-12.

18. Nimura M. Neurofibromatosis. Rinsho Derma. 1973;15:653-663.

19. Mansukhani SA. Familial Schwannomatosis: A Diagnostic Challenge. Journal of Clinical and Diagnostic Research. 2017.

20. Adeloye A. Coexistence of acromegaly and neurofibromatosis in a Nigerian. East African medical journal. 1979;56(1):38-39.

21. Ademiluyi SA, Ijaduola TGA. Neurofibromatosis in Nigerian children.J Natl Med Assoc. 1988;80(9):10141017 .

22. Ademiluyi SA, Sowemimo GO, Oyeneyin JO. Surgical experience in the management of multiple neurofibromatosis in Nigerians. West African journal of medicine. 1989;8(1):59-65.

23. Ahmed H, Falope ZF. An unusual association of a rare variant of Friedreich's ataxia with type-I neurofibromatosis in a Nigerian Fulani family: a 5-year follow-up study. West African journal of medicine. 1999;18(2):133-138.

24. Odebode TO, Afolayon EAO, Adigun IA, Daramola OOM. Clinicopathological study of neurofibromatosis type 1: an experience in Nigeria. International journal of dermatology. 2005;44:116-120.

25. Okoromah CN, Adegbola TA, Ojuola OI. Neurofibromatosis with malignant transformation presenting as an emergency in a Nigerian child: a case report. The Nigerian postgraduate medical journal.2005;12(1):49-52.

26. Asuquo M, Nwagbara V, Nnoli M, Ashindoitiang J, Ugbem T, Akpan S. Neurofibrosarcoma: case series in south-south Nigeria.International Journal of Medicine. 2017;5(1):79.

27. Adeleye AO, Okolo CA, Akang EE, Adesina AM. Cerebral pleomorphic xanthoastrocytoma associated with NF1: an updated review with a rare atypical case from Africa. Neurosurgical Review.2011;35(3):313-319.

28. Ibrahim A, Asuku ME. Neurofibromatosis. The New England journal of medicine. 2011;365:21.

29. Isa SE, Akindele S, Daniyam C, Iroezindu M, Agaba A, Agbaji O. An HIV-1 Infected Patient with Neurofibromatosis Type 1: A Case Report.Nigerian Health Journal. 2012;12(2):52-53. 
30. Azeke AT, Imasogie DE. A review of histologically diagnosed neurofibromatosis; an institution based study spanning a decade.SSRG International Journal of Medical Science 2018;5(6):1-4.

31. Anyabolu EN. Lumbar plexiform neurofibroma, short stature, and kyphoscoliosis in neurofibromatosis-1: A Rare entity. Sahel Medical Journal. 2019;22(3):153.

32. Nyandaiti YW, Tahir C, Nggada HA, Ndahi AA. Clinico-pathologic presentation and management of neurofibromatosis type 1 (Von Recklinghausen's) disease among north-eastern Nigerians: A six year review. . Niger Med J. 2009;50:80-83.

33. National Institute of Health (NIH). Neurofibromatosis. NIH Consensus Statement Online. Vol 6 Online National Institute of Health; 1987:1-19.

34. National Institutes of Health. National Institutes of Health Consensus Development Conference Statement: neurofibromatosis. Bethesda, Md., USA, July 13-15, 1987. Neurofibromatosis. 1988;1(3):172-178.

35. Bergqvist C, Servy A, Valeyrie-Allanore L, Ferkal S, Combemale P, Wolkenstein P; NF France Network. Neurofibromatosis 1 French national guidelines based on an extensive literature review since 1966. Orphanet Journal of Rare Diseases. 2020 Feb 3;15(1):37. doi: 10.1186/s13023-020-1310-3.

36. Yusuf A, Pillai A, Menon S, Panikar D. Massive spontaneous hemothorax, giant intrathoracic meningocele, and kyphoscoliosis in neurofibromatosis type 1. Journal of Surgical Technique and Case Report. 2014;6(1):33.

37. Elsayed SM, Fahmy N, Gamal R, et al. Neurofibromatosis type 1 and multiple sclerosis: Genetically related diseases. Egyptian Journal of Medical Human Genetics. 2017;18(3):295-298.

38. Boley S, Sloan JL, Pemov A, Stewart DR. A Quantitative Assessment of the Burden and Distribution of Lisch Nodules in Adults with Neurofibromatosis Type 1. Investigative Opthalmology 8 Visual Science. 2009;50(11):5035-5043.

39. Ragge NK, Falk RE, Cohen WE, Murphree AL. Images of Lisch nodules across the spectrum. Eye. 1993;7:95-101.

40. Cruciani F, Carmen Piraino D, Albanese G, Rahimi S, Abdolrahimzadeh B. Neurofibromatosis: an update of ophthalmic characteristics and applications of optical coherence tomography. Clinical ophthalmology. 2016:851.

41. Coy S, Rashid R, Stemmer-Rachamimov A, Santagata S. An update on the CNS manifestations of neurofibromatosis type 2. Acta Neuropathologica. 2019;139(4):643-665.

42. Fried I, Tabori U, Tihan T, Reginald A, Bouffet E. Optic pathway gliomas: a review. CNS Oncol 2013;2(2):143-159.

43. Olubunmi OA. Misdiagnosis of tuberous sclerosis in a Nigerian girl: A case report and review of literature. Ann Afr Med 2010;9:95-101.

44. Elechi EN, Etawa US. Solitary lumbar neurofibroma in a patient with neurofibromatosis. Tropical doctor. 1994;24(4):174 doi: 10.1177/004947559402400415..

45. Asuquo ME. Neurofibrosarcoma - complicating neurofibromatosis-I: case report and review of relevant literature. The Nigerian postgraduate medical journal. 2005;12(3):233-236.

46. Legbo JN, Shehu BB, Malami SA. Malignant peripheral nerve sheath tumour associated with von Recklinghausen's disease: case report.East African medical journal. 2005;82(1):47-49.

47. Shehu BB, Hassan I. Cervicothoracic arachnoid cyst in a patient with neurofibromatosis: case report. East African medical journal.2006;83(9):515-517. 
48. Etiuma AO, Bassey OO, Asuquo E, Ebughe G. Multiple Neurofibrosarcoma. Nig J Surgery. 2007;13(12):29-31.

49. Ekpe EE. Malignant transformation in von recklinghausen disease in a rural farmer presenting as huge metastatic soft tissue chest wall tumour: a case report. West African journal of medicine.2014;33(1):77-79.

50. Nasir AA. Re: 'Diffuse ganglioneuromatosis and plexiform neurofibroma of the urinary bladder: an uncommon cause of severe urological disease in an infant'. J Pediatr Urol. 2013 Apr; 9(3):e131-e133. Journal of pediatric urology. 2014;10(1):198.

51. Yahya H, Sani H. Eruptive neurofibromas in pregnancy. Annals of African medicine. 2020;19(2):150-152.

52. Mautner VF, Hartmann M, Kluwe L, Friedrich RE, Fünsterer C. MRI growth patterns of plexiform neurofibromas in patients with neurofibromatosis type 1. Neuroradiology. 2006;48(3):160-165.

53. Eldridge R, Denckla MB, Bien E, et al. Neurofibromatosis type 1 (Recklinghausen's disease). Neurologic and cognitive assessment with sibling controls. American journal of diseases of children.1989;143(7):833-837.

54. Bergqvist C, Servy A, Valeyrie-Allanore L, Ferkal S, Combemale P, Wolkenstein P. Neurofibromatosis 1 French national guidelines based on an extensive literature review since 1966. Orphanet Journal of Rare Diseases. 2020;15(1).

55. Wolsey DH, Larson SA, Creel D, Hoffman R. Can Screening for Optic Nerve Gliomas in Patients With Neurofibromatosis Type I Be Performed With Visual-Evoked Potential Testing? Journal of American Association for Pediatric Ophthalmology and Strabismus.2006;10(4):307-311.

\section{Figure Legend}

Figure 1: Prevalence of NIH diagnostic signs (criteria) among patients presenting to ophthalmology clinic with Neurofibromatosis in a black African population

Figure 2: Distribution of plexiform neurofibromas in patients presenting with Neurofibromatosis in a black African population

Figure 3: Pigmentation in black African patients with NF1.

a-b : Café sans laît (CSLM) macules some of which are almost imperceptible (black arrow) against the dark skinned complexion.

c : Café au laît macules on the buttocks of 10 year old boy with NF1. A hypopigmented macule (laît) is indicated in this photograph, using double black arrows.

d : Hyperpigmented, somewhat hypertrophic, CALMs on the scalp of a 10-year-old boy with NF1. The scalp is an unusual location for CALMs in Caucasians and is usually spared (Friedmann, J.M., 2002) ${ }^{4}$

Figure 4- Hematoxylin and eosin stained sections- Histopathological findings of a typical plexiform neurofibroma from an eyelid nodule in an 11 year old female

(X40) Skin with enlarged dermal nerves, containing large nerve bundles.

B and C. (X100) Irregularly contoured and enlarged nerves.

D. (X400) Enlarged nerve with a cellular matrix containing fibroblasts, Schwann cells, collagen and mucin in the involved nerve.

Figure 5: a. Plexiform neurofibroma of the nose in a 12-year-old male.

b. Orbito-cranial plexiform neurofibroma causing proptosis in a 15-year-old female (below) notice also the plexiform neurofibroma of the left pinna and hyperpigmented macules. 
c,d : Pseudoarthrosis and deformity of interphalangeal joint of $5^{\text {th }}$ digit (digiti minimi quintis), of 15-year-old female with NF 1. Note: notice also unusual freckling (hyperpigmented macules) on both palms, this is also an rare site for CALMs in Caucasians (Friedmann, J.M., 2002)

e . Bone dysplasia causing lateral bowing of the tibia (above) notice the cutaneous neurofibroma on the contralateral leg $\mathbf{f}$. Absence of the left lesser wing of the sphenoid (below) in the same 15-year-old female with NF1

Figure 6: a: Lisch nodules in the iris appearing as multiple raised flat-topped yellow spots in 12-year old female (seen without slit lamp) b: Lisch nodules seen with magnification, using slit lamp in a 45-year old woman, first degree relative. Notice the irregular but well circumscribed borders, this woman also has a nasal pterygium.

Figure 6 c,d: Fundus photograph showing bilateral optic atrophy in a 17-year-old male presenting with clinical features of neurofibromatosis 1, Brain MRI (Figure 9) showed bilateral optic-hypothalamic pathway glioma

Figure 6 e,f: Optic-hypothalamic pathway glioma in the 17 year old male presenting with neurofibromatosis and bilateral optic atrophy

Note : Abnormal hyperintensity and expansion of the optic tracts (6e ) and enlargement of the optic chiasm (6f ), worse on the left, are shown on low-field magnetic resonance imaging (0.4 Tesla) of the brain.

Table 1: Neuro-imaging diagnoses of patients with neurofibromatosis type1, showing recommendations for multidisciplinary management.

\begin{tabular}{lll}
\hline & Clinical Presentation & Neurc \\
\hline $10 \mathrm{y}, \mathrm{M}$ & Redness and loss of vision OD, papilloedema OS & Brain \\
$14 \mathrm{y}, \mathrm{M}$ & Upper lid mass OS, Poor school performance & Orbit \\
$15 \mathrm{y}, \mathrm{F}$ & Cranio-facial mass with intracranial extension, OS & Orbit \\
$17 \mathrm{y}, \mathrm{M}$ & Poor vision x 10 years, Poor school performance, Optic atrophy OU, Mother had cutaneous NF nodules & Brain \\
$18 \mathrm{~m}, \mathrm{M}$ & Upper back mass with scoliosis & MRI \\
$7 \mathrm{y}, \mathrm{M}$ & Right sided cranio-facial mass since birth, short stature & Orbit \\
\hline
\end{tabular}

MRI: Magnetic resonance imaging; CT: computed tomography; m: months; y: years; OD: right eye; OS: left eye; CNS: central nervous system; NF: neurofibroma 


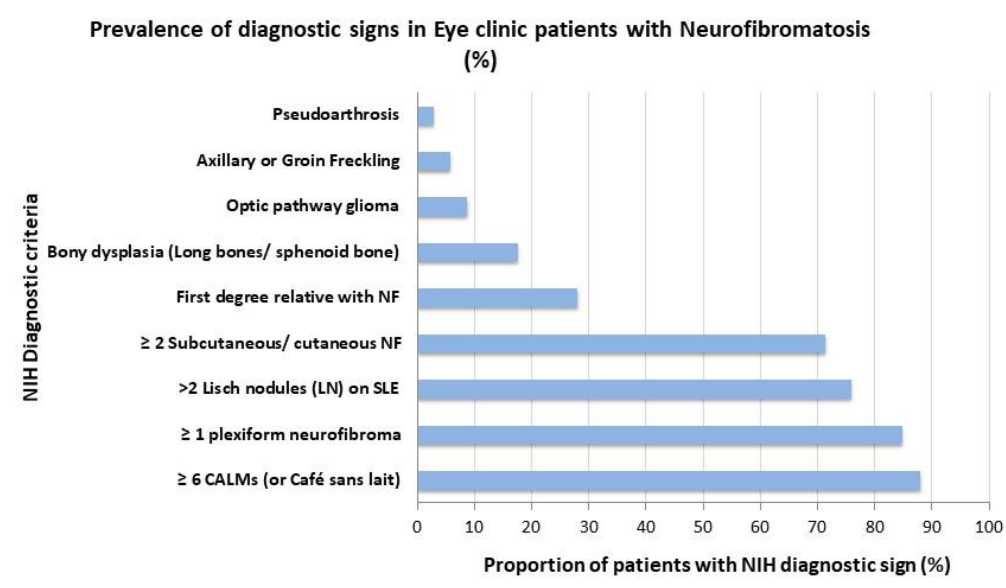

*CALMs, Café au laît macules; SLE, Slit-lamp examination; NF, Neurofibroma

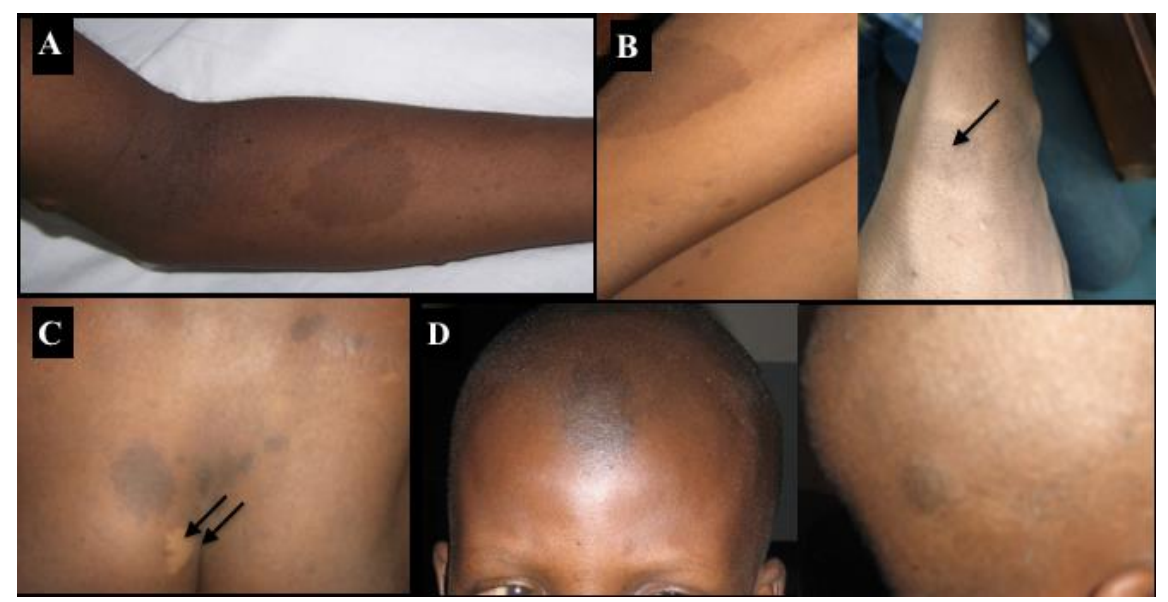


Distribution of plexiform neurofibromas at presentation ( $\mathrm{N}=28$ )
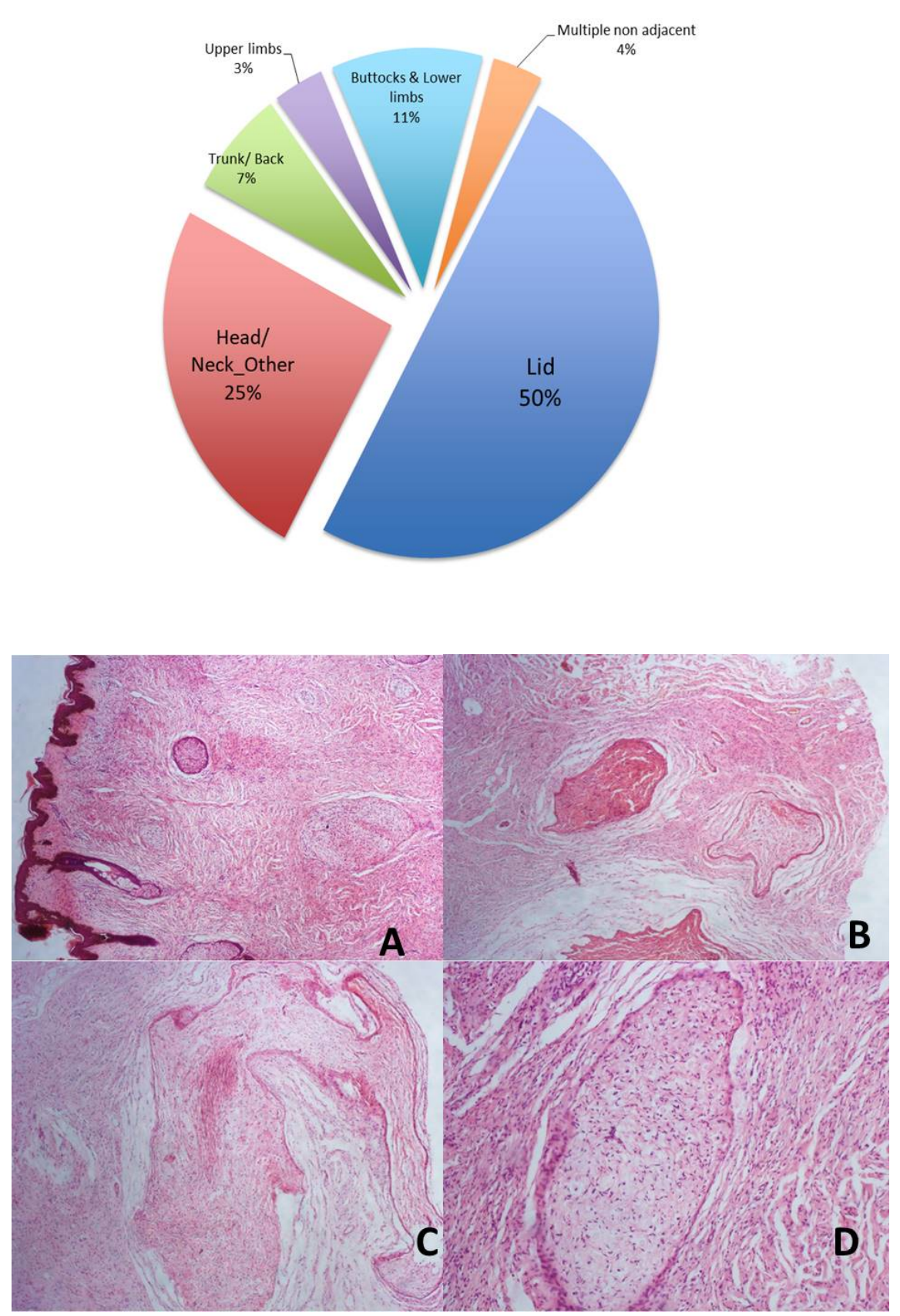

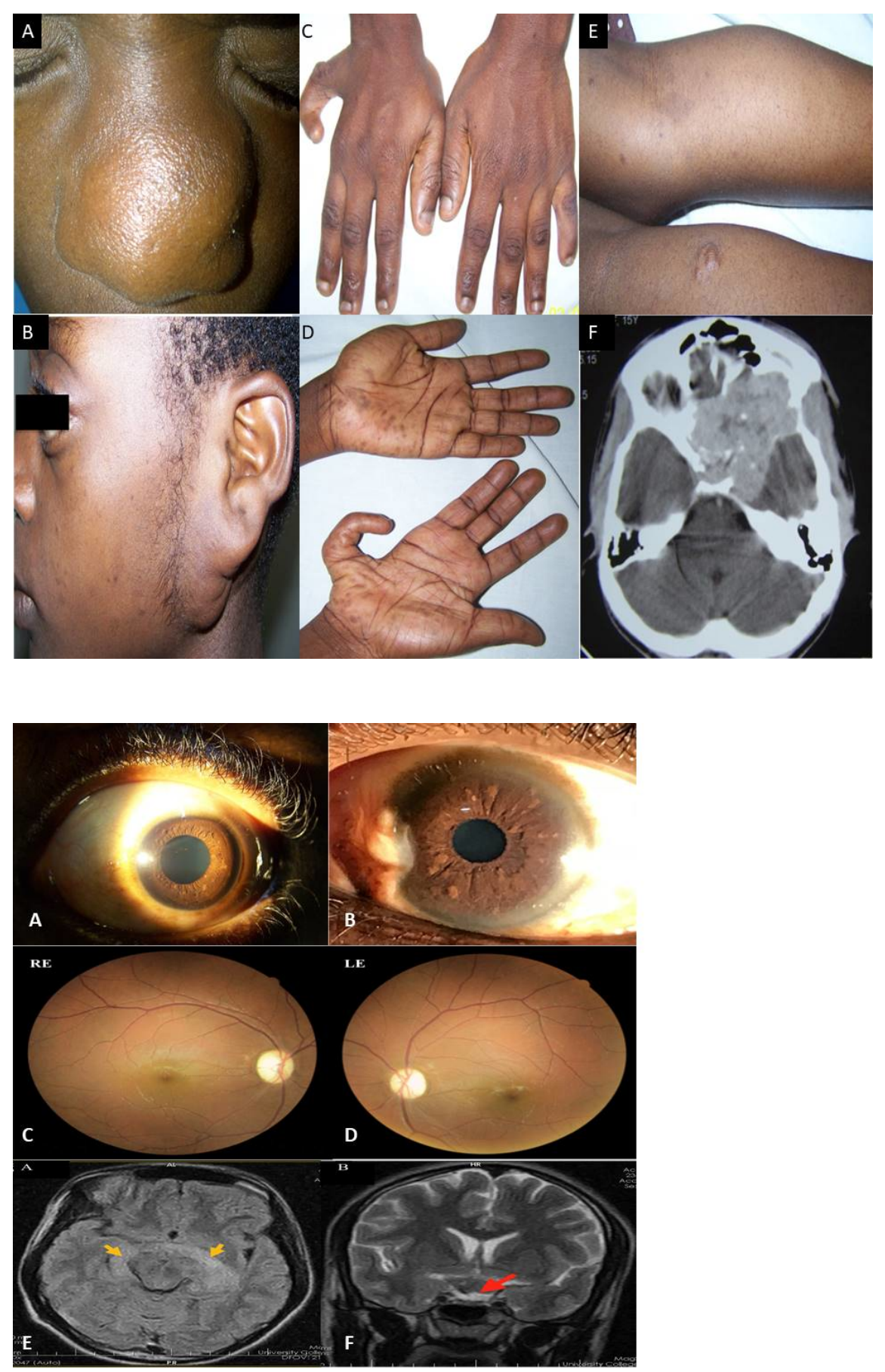